This is the post print of,

LORENTE S. , VIVES J. \& LOSILLA J. M. (2017) Instruments to assess the patient comfort during hospitalization: a psychometric review protocol. Journal of Advanced Nursing, 73 (3), 735-741. doi: $10.1111 /$ jan.13180

\title{
INSTRUMENTS TO ASSESS THE PATIENT COMFORT DURING \\ HOSPITALIZATION: A PSYCHOMETRIC REVIEW PROTOCOL
}

Sonia LORENTE, Jaume VIVES, Josep Maria LOSILLA

Universitat Autònoma de Barcelona

Sonia LORENTE, Psychologist, Department of Psychobiology and Methodology of Health Science Area of Behavioral Science Methodology, Universitat Autònoma de Barcelona, UAB; Paediatric nurse, General Paediatric Ward, Hospital de Terrassa, Consorci Sanitari de Terrassa; Jaume VIVES; Josep Maria LOSILLA, PhD, Lecturers, Department of Psychobiology and Methodology of Health Science Area of Behavioral Science Methodology, Universitat Autònoma de Barcelona, UAB.

The authors declare no conflict of interest. Protocol supported with the Grant PSI2014-52962-P, Spanish Ministry of Economy and Competitiveness (July, 2015). Correspondence concerning this article should be addressed to Sonia Lorente, Department of Psychobiology and Methodology of Health Science Area of Behavioral Science Methodology, Universitat Autònoma de Barcelona, UAB. E-mail: sonia.lorentes@ecampus.uab.cat 


\section{IMPACT STATEMENT}

Measuring the patient experience is essential to improve the quality of care provided in the different health settings. Enhancing the patient comfort as a holistic experience is related to quicker discharges, fewer readmissions, increased patient satisfaction and stronger costbenefit ratios for the institution. 


\section{ABSTRACT}

Aim: To analyse the psychometric properties, outcomes and utility of instruments measuring the patient comfort during hospitalization. Background: While there are numerous systematic reviews assessing the psychometric properties of health care instruments, none of them is devoted to evaluate the psychometric properties of instruments measuring comfort, which is considered an indicator of quality in health care and is associated to quicker discharges, increased patient satisfaction and stronger cost-benefit ratios for the institution.

Design: Systematic review. Methods: The review will follow the Preferred Reporting Items for Systematic Reviews and Meta-Analyses Guidelines (PRISMA). Search will be performed on MEDLINE, CINAHL, PsycINFO, Web of Knowledge, ProQuest Thesis\&Dissertations, and grey literature, and will be focused questionnaires measuring the patient comfort as a holistic experience in any health care setting. The assessment will take into account the theoretical model upon which the instruments are built, will evaluate the psychometric properties of each study according to the COnsensus-based Standards for the selection of health status Measurement INstruments (COSMIN), and will include the assessment of the quality of instruments outcomes and their cost-efficiency, acceptability and educational impact. The review protocol was registered in PROSPERO, CRD42016036290, and was supported by the Grant PSI2014-52962-P, Spanish Ministry of Economy and Competitiveness (July, 2015). Discussion: The results of our psychometric review will categorise the instruments measuring the patient comfort according to their psychometric properties, methodological quality, outcomes and utility, in order to improve the quality of health care provided and the institution's benefits. Keywords: Psychometric Review, Patient comfort, Hospitalization, Cost-efficiency, Comfort assessment, Nursing care. 


\section{SUMMARY STATMENT: WHY THIS REVIEW IS NEEDED?}

- There are no systematic reviews nor meta-analyses of health instruments measuring the patient comfort during the hospitalization; comfort is considered a direct indicator of quality in any health care.

- Assessing the psychometric properties, the outcomes and the utility of the instruments measuring the patient comfort is essential in order to improve the quality of care. 


\section{INSTRUMENTS TO ASSESS THE PATIENT COMFORT DURING \\ HOSPITALIZATION: A PSYCHOMETRIC REVIEW PROTOCOL}

\section{Background}

Measuring the patient experience is essential to improve the quality of care provided in the different health settings (WHO 2000, 2004,2013). To date, different health care instruments have been developed aiming to assess the patient experience to enhance the quality of care. As reliable and valid instruments are essential in health care research and clinical practice, numerous systematic reviews have been developed to assess the methodological quality of these instruments. These reviews include instruments measuring health related quality of life, pain or fatigue, to name a few (Mokkink et al. 2009). Nevertheless, there are no systematic reviews nor meta-analyses of health instruments measuring the patient comfort during the hospitalization, being comfort a direct indicator of quality in health care (NQMC 2002; Peterson \& Bredow 2013). As enhancing the patient comfort is associated to quicker discharges, fewer readmissions, increased patient satisfaction and stronger cost-benefit ratios for the institution (Kolcaba 2001; Peterson \& Bredow 2013), is essential for clinicians, researchers and institutions to know how the instruments assessing comfort perform. With this purpose, their psychometric properties, their utility and their outcomes should be assessed, as the health instruments need to have high utility as well as they must be valid and reliable. Otherwise there is a serious risk of biased results that may lead to wrong results (Keszei et al. 2010; Streiner \& Norman 2008; Terwee et al. 2007)

Conceptual Framework

Patient comfort is considered an individualized and holistic experience, source of patient satisfaction and well-being. The concept of comfort was framed within the Theory of Comfort, by Kolcaba, and was defined theoretically as "the state of having met basic human 
needs for ease, relief and transcendence" in four contexts (physical, psychospiritual, sociocultural and environmental) (Kolcaba, 1991: 240) (Kolcaba \& Kolcaba 1991; Kolcaba 1992; Kolcaba 1995). Within this context, the General Comfort Questionnaire (GCQ) is likely the first instrument specifically developed to measure the patient comfort as a holistic experience, in the four contexts, and registered as a multidisciplinary outcome indicator of quality in health care in the National Quality Measures Clearinghouse (Kolcaba 1992; NQMC 2002; Peterson \& Bredow 2013). Since then, a large range of instruments to assess the patient comfort have been developed, adapted or validated.

Aims

Due to the lack of previous systematic reviews assessing the comfort instruments performance, this study aims to examine the psychometric properties, outcomes and utility of each questionnaire measuring the patient comfort, and discuss the practical and the research applications of these instruments in the current health context.

1. Identify the health instruments measuring the patient comfort as a holistic experience during hospitalization.

2. Examine the psychometric properties of each instrument assessing their reliability, and validity,

3. Examine the outcomes quality including the reproducibility, responsiveness, floorceiling effects and interpretability.

4. Examine the utility of each instrument assessing their cost efficiency, acceptability and educational impact in different health care settings.

5. Classify the different instruments according to their psychometric properties, outcomes and utility. 


\section{METHODOLOGY}

This psychometric review will follow the Preferred Reporting Items for Systematic Reviews and Meta-Analyses Guidelines (PRISMA) (Liberati et al. 2009; Moher et al. 2009). This study has been registered in PROSPERO, an international database of prospectively registered systematic reviews in health and social care (protocol registration number CRD42016036290), available at

http://www.crd.york.ac.uk/PROSPERO/display record.asp?ID=CRD42016036290

Search strategy

We aim to identify published instruments measuring the patient comfort during the hospitalization. We will define different combinations of keywords (using Mesh and other thesauruses, where available), in relation to the concept (e.g., comfort, theory), the setting (hospitalization or admission) and the instruments (e.g. questionnaires, scales). The following databases will be included: Medical Literature Analysis and Retrieval System (MEDLINE), by ProQuest, Cumulative Index to Nursing and Allied Health Literature (CINALH), by EBSCOhost, Psychological Information (PsycINFO), by APA PsycNET, Thesis \& Dissertations, by ProQuest, and ISI Web of Knowledge (WoS, Web of Science CORE), by Thomson Reuters. To include grey literature, we will also search records in Google, and will review up to 400 links. The search will be limited by population (humans), by time (1990 to 2015) and by language (English). In addition, search alerts in CINALH \& PsycINFO will be set. The Table 1 shows an example of this search strategy.

Inclusion criteria

Time frame 
From 1990 (since the first instrument measuring the patient comfort seems to have been developed in 1992, framed within the Theory of Comfort, by Kolcaba) to 2015 .

\section{Study type}

Studies developing or validating questionnaires and/or scales measuring the holistic patient comfort during the hospitalization. Protocols and guidelines, as well as those studies exclusively qualitative designed, will be excluded.

Age group

We will include the whole range of ages (newborns, toddlers, children, teenagers, young adults, middle-age adults and elderly people).

\section{Context}

We will evaluate instruments measuring the comfort in different healthcare settings where the patient is hospitalized due to acute illnesses (e.g. pneumonia, urinary tract infection), chronic pathologies (e.g. psychiatric illnesses, dementia), surgical interventions and labour issues. Therefore, the following settings will be included: general paediatric wards, general adult wards, the delivery room and the maternity ward, the operating room, the Intensive Care Unit (ICU), the Paediatric Intensive Care Unit (PICU), the Neonatal Intensive Care Unit (NICU), the postoperative area, the psychiatric ward and the hospice.

\section{Instruments}

We will include instruments developed with scales and/or subscales with close-ended answer items as Likert, Visual Analogic Scales (VAS) and/or Numerical Rating Scales (NRS) specifically designed for measuring the patient comfort as a holistic experience during the hospitalization. Instruments measuring the comfort related to screening or diagnostic tests will be excluded (e.g. colonoscopy, bronchoscopy, angiography, mammography, injections, 
biopsies, cystoscopy, cytology, fertility treatments), as well as those instruments assessing the comfort exclusively by physiological parameters (i.e. heart rate and/or blood pressure) or measuring the effect of a specific intervention (e.g. warm blanket vs. classical blanket or midazolam vs. fentanyl).

\section{Data extraction}

A reviewer will apply the inclusion criteria to all titles and abstracts. If no decision can be taken based solely on title and abstract alone, the full paper will be retrieved. Authors of eligible studies will be contacted to provide missing or additional data if necessary. Full-text inclusion criteria will be checked independently by two review authors. Discrepancies will be resolved through discussion (with a third author where necessary).

A pre-piloted form will be used to extract data from the included studies in order to assess the study quality and to synthesize the evidence. Extracted information of each selected instrument will include: general information (author, year, country of origin and papers); instrument detail (outcome measures, purpose/use, number of items, response categories, scale design, type of patients); utility characteristics (theoretical/conceptual framework, validity tests conducted and results, reliability tests conducted and results, response rate, sample size, setting, respondents population and demographics, ease and usefulness of interpretation, cost- efficiency, level of expertise required for scoring, acceptability, time required to completion, mode of administration, acceptability by managers and users, educational impact). Two review authors will extract data independently, and discrepancies will be identified and resolved through discussion (with a third author where necessary).

Strategy for data analysis

We will initially categorise the instruments according to the theoretical model used to design and validate the instrument: reflective vs. formative. To decide whether the model is 
reflective or formative we will take into account the theoretical and practical considerations detailed in the Table 2 (Coltman et al. 2008). Secondly, we will apply the COSMIN checklist in four steps (see Figure 1), and we will use the four-point scoring system (excellent, good, fair or poor) (Mokkink et al. 2010; Mokkink et al. 2012; Terwee et al. 2011; Terwee et al. 2012). We will also apply the Quality Criteria for Measurement Properties for assessing the design, the methods and the outcomes of each instrument. These criteria consist of assessing the content validity, the internal consistency, the criterion validity, the construct validity, the reproducibility, the responsiveness, the floor and ceiling effects and the interpretability (Terwee et al. 2007).

-Insert Table 2 here or near here-

-Insert Figure 1 here or near here-

Finally, we will assess the cost-efficiency, the acceptability and the educational impact of each instrument to discuss their utility in the real practice world, according to Van der Vleuten's Utility Index Matrix (Van der Vleuten, 1996, cited in Beattie, Lauder, Atherton, \& Murphy 2015).

Strategy for data synthesis

Where applicable, we will use the general framework and specific tools outlined in the ESRC Guidance on the Conduct of Narrative Synthesis in Systematic Reviews (Popay et al. 2007). The extracted information by using the pre pilot form and the results of the whole process will be showed in tables as well as they will be synthesized in a narrative way.

Validity and reliability

To minimize the risk of bias during the methodological analysis of each instrument we will apply the COnsensus-based Standards for the selection of health status Measurement 
INstruments (COSMIN) (Mokkink, Terwee, Knol, et al. 2010; Mokkink et al. 2012; Terwee et al. 2012). With the same purpose, and to improve the quality of reporting of results of evaluation of health care instruments, we will apply the Quality Criteria for Measurement Properties (Terwee et al. 2007).

Ethical considerations

Non-specific ethical issues 


\section{DISCUSSION}

This psychometric review will contribute to categorise the instruments measuring the patient comfort according to their psychometric properties, their outcomes and their clinical and research utility. Concerning our results, different practical issues should be taken into account. First, assessing the psychometric properties of these instruments, as validity and reliability, would be helpful either for those researchers interested in improving the quality of these health care instruments, or for those clinicians interested in measuring the patient comfort during the hospitalization. Second, assessing the quality of outcomes, as reproducibility, responsiveness, floor-ceiling effects and interpretability, would be helpful for those researchers and clinicians interested in identifying and applying the best quality questionnaires measuring comfort. Third, assessing the utility of each instrument, as the costefficiency, the acceptability and the educational impact, would be helpful for clinicians and institutions to choose the most efficient instrument according the health care setting, patient and pathology. Furthermore, choosing the most suitable and efficient questionnaire may minimize the risk of getting incomplete questionnaires because of the large amount of time needed for their administering or to the important cognitive effort required from some patients with certain circumstances. Finally, we will discuss the practical and clinical applications related to the cost-efficiency as the sample needed to achieve the adequate reliability, the professional expertise required for administering the questionnaires, and the institution cost-benefits resulting from the research in this field.

Limitations

The present review will only include studies published in English. 


\section{Conflict of interest}

The authors declare no conflict of interest. The authors have no financial relationships relevant to this article to disclose.

Funding

This research is supported by Grant PSI2014-52962-P, from the Spanish Ministry of Economy and Competitiveness. The approval to support this research by this grant has been obtained in July, 2015

Authors' contribution

All authors conceptualized and designed this study. SL devised search strategies, piloted the inclusion criteria and drafted the manuscript. JV and JML provided direction of the design, piloted the inclusion criteria, provided direction of the data extraction and analysis, and critically revised the manuscript draft. All authors approved the final manuscript. 
References

Beattie, M. et al., 2015. Instruments to measure patient experience of health care quality in hospitals: a systematic review. Systematic Reviews, 3(1): 1-4.

Coltman, T. et al., 2008. Formative versus reflective measurement models: Two applications of formative measurement. Journal of Business Research, 61(12): 1250-1262.

Keszei, A., Novak, M. \& Streiner, D.L., 2010. Introduction to health measurement scales. Journal of Psychosomatic Research, 68(4): 319-323.

Kolcaba, K., 1992. Holistic comfort: operationalizing the construct as a nurse-sensitive outcome. Advances in nursing science, 15(1): 1-10.

Kolcaba, K., 1995. The art of comfort care. Journal of Nursing Scholarship, 27(4): 287-289.

Kolcaba, K., 2001. Evolution of the mid range theory of comfort for outcomes research. Nursing Outlook, 49(2): 86-92.

Kolcaba, K. 2013, " Comfort", in Peterson, S.J. \& Bredow, T.S. (Third edit) Middle Range Theories. Application to nursing research pp.193-209.

Kolcaba, K. \& Kolcaba, R., 1991. An analysis of the concept of comfort. Journal of Advanced Nursing, 16(11): 1301-1310.

Liberati, A. et al., 2009. The PRISMA Statement for Reporting Systematic Reviews and Meta-Analyses of Studies That Evaluate Health Care Interventions : Explanation and Elaboration. Annals of Internal Medicine, 151(4): W65-W94.

Moher, D. et al., 2009. Preferred Reporting Items for Systematic Reviews and MetaAnalyses: The PRISMA Statement. Annals of Internal Medicine, 151(4): 264-269. 
Mokkink, L.B. et al., 2009. Evaluation of the methodological quality of systematic reviews of health status measurement instruments. Quality of Life Research, 18(3): 313-333.

Mokkink, L.B. et al., 2010. The COSMIN checklist for evaluating the methodological quality of studies on measurement properties: a clarification of its content. Medical Research Methodology, 10(1): 1-8

Mokkink, L.B. et al., 2012. The COSMIN checklist manual. Available at http://www.cosmin.nl (Last access on 28 July 2016)

Popay, J. et al., 2007. Guidance on the Conduct of Narrative Synthesis in Systematic Reviews. Available at https://www.researchgate.net/publication/233866356 (Last access on 28 July 2016)

Streiner, D.L. \& Norman, G.R., 2008. Health Measurement Scales: A practical guide to their development and use Fourth Edit., OUP Oxford.

Terwee, C.B., Mokkink, L.B. \& Patrick, D.L., 2011. COSMIN checklist with 4-point scale. Available at http://www.cosmin.nl( Last access on 28 July 2016)

Terwee, C.B. et al., 2007. Quality criteria were proposed for measurement properties of health status questionnaires. Journal of Clinical Epidemiology, 60(1): 34-42.

Terwee, C.B. et al., 2012. Rating the methodological quality in systematic reviews of studies on measurement properties: A scoring system for the COSMIN checklist. Quality of Life Research, 21(4): 651-657.

World Health Organization, 2000. Strategies for assisting health workers to modify and improve skills: developing quality health care-a process of change. Issues in Health Services. Available at http://www.who.int/en/ (Last access on 28 July 2016) 
World Health Organization, 2004. Standards for Health Promotion in Hospitals. Available at http://www.who.int/en/ (Last access on 28 July 2016)

World Health Organization, 2013. Health 2020: A European Policy Framework and Strategy for the 21st Century. Available at http://www.who.int/en/ (Last access on 28 July 2016) 
Table 1. Search strategy in CINALH \& PsycINFO

\section{CINALH (EBSCOhost)}

TI (comfort* OR discomfort* OR uncomfort*) AND AB (theor* OR questionnaire* OR instrument* OR measure* OR scale* OR assessment* OR hospital* OR admission*) NOT (chair* OR ergonomic* OR thermal* OR cloth*) 1990 to 2015 Filters; Humans, English

\section{PSYCINFO (APA PsycNET)}

Title: comfort* OR discomfort* OR uncomfort* AND Abstract: theor* OR questionnaire* OR instrument* OR measure* OR scale* OR assessment* OR hospital* OR admission* NOT Any Field: chair* OR ergonomic* OR thermal* OR cloth* AND Language: English AND Population Group: Human AND Year: 1990 To 2015 
Figure 1. The four step procedure to complete the COSMIN checklist

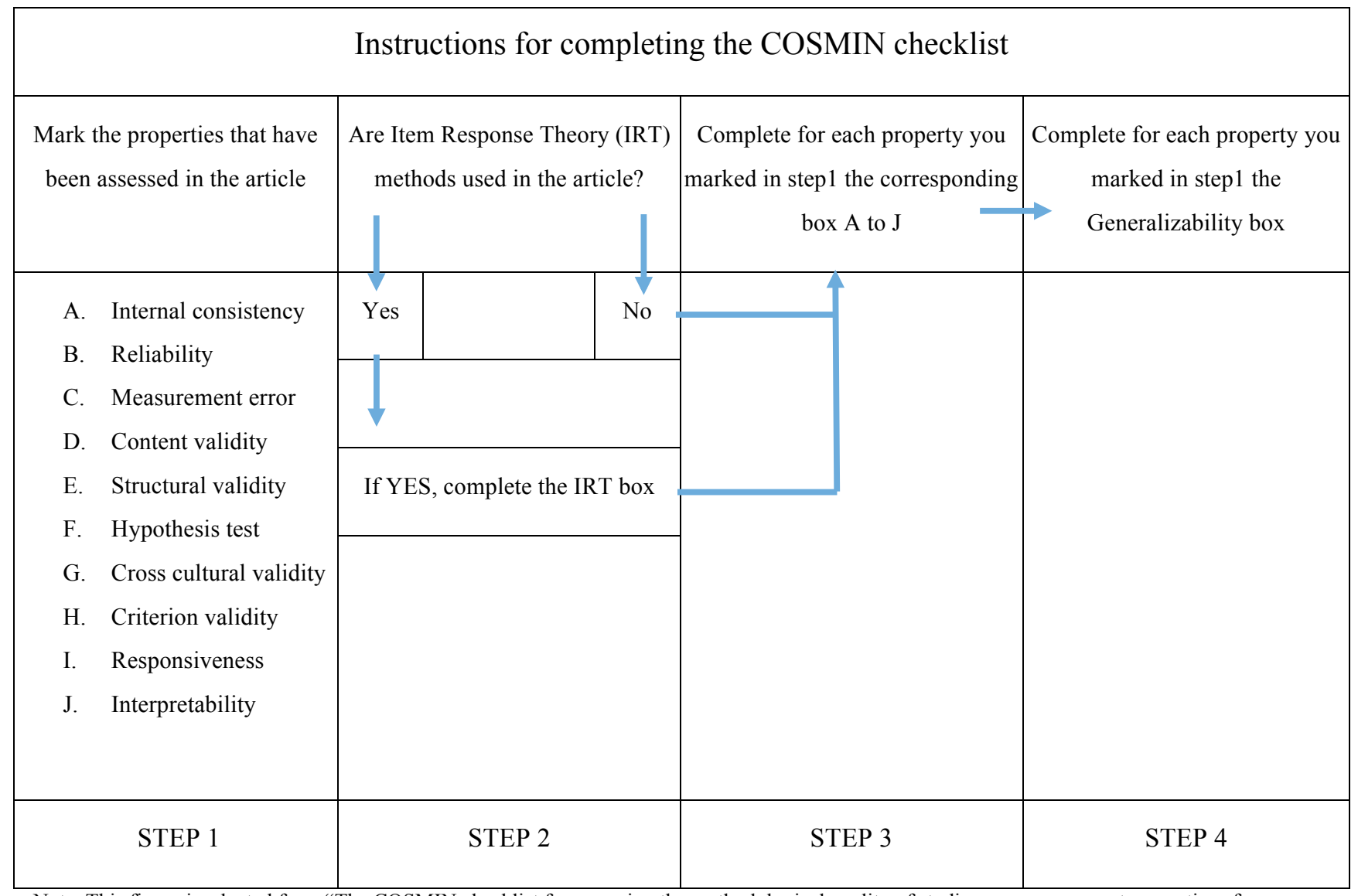

Note. This figure is adapted from "The COSMIN checklist for assessing the methodological quality of studies on measurement properties of health status measurement instruments: an international Delphi study", by Mokkink, Terwee, Knol, et al., 2010, Quality of Life Research, 19, p. 544. 
Table 2. A framework for assessing reflective and formative models.

\begin{tabular}{lll}
\hline Considerations & Reflective model & Formative model \\
\hline
\end{tabular}

\section{Theoretical considerations}

1.Nature of construct

Latent construct exists $\checkmark \quad$ Latent construct exists independent of the
measures used

2.Direction of causality between Causality from construct to items items and latent construct
$\checkmark \quad$ Variation in the construct causes variation in the item measures
$\checkmark \quad$ Variation in item measures does not cause variation in the construct

3. Characteristics of items used to Items are manifested by the construct measure the construct
$\checkmark \quad$ Items share a common theme
$\checkmark \quad$ Items are interchangeable
$\checkmark$ Adding or dropping an item does not change the conceptual domain of the construct

Latent construct is formed

$\checkmark \quad$ Latent constructs is a combination of its indicators

Causality from items to construct

$\checkmark \quad$ Variation in the construct does not cause variation in the item measures

$\checkmark \quad$ Variation in item measures causes variation in the construct

Items define the construct

$\checkmark \quad$ Items need not share a common theme

$\checkmark \quad$ Items are not interchangeable

$\checkmark \quad$ Adding or dropping an item may change the conceptual domain of the construct

\section{Empirical considerations}

4.Item intercorrelation

4. Item intercorrelation construct antecedents and consequences

6.Measurement error and collinearity
Items should have high positive intercorrelations

$\checkmark \quad$ Empirical tests: assessing internal consistency and reliability by Cronbach alpha, average variance extracted and factors loadings (e.g. from common or confirmatory factor analysis)

Items have a similar sign and significance of relationship with the antecedents/consequences as the construct

$\checkmark \quad$ Empirical tests: establishing content validity by theoretical considerations, assessing convergent and discriminant validity empirically.

Identifying the error in items is possible

Empirical test: identifying and extracting measurement error by common factor analysis
Items can have any pattern of intercorrelation but should possess the same directional relationship

$\checkmark$ Empirical tests: no empirical assessment of indicator reliability is possible; various preliminary analyses are useful to check directionality between items and construct

Items may not have similar significance of relationships with the antecedents/consequences as the construct

$\checkmark \quad$ Empirical tests: assessing nomological validity by using a MIMIC model, and/or structural linkage with another criterion variable

Identifying the error is not possible if the formative measurement model is estimated in isolation

$\checkmark$ Empirical test: using the vanishing tetrad test to determine if the formative items behave as predicted. Collinearity should be ruled out by standard diagnostics such as the condition index

\footnotetext{
Note: This table is adapted from "Formative versus reflective measurement models: Two applications of formative measurement", by

Coltman et al., 2008, Journal of Business Research, 61, p. 1252
} 